\section{Compression in mixed ulcers}

\section{Giovanni Mosti}

Angiology Department, Barbantini Clinic, Lucca, Italy

Peripheral arterial occlusive disease (PAOD) is reported in about $15-20 \%$ of patients with venous leg ulcers (VLU). In such cases compression therapy is considered a contraindication when ankle-brachial pressure index (ABPI) is $<0.8$.

Aim of this short paper is to briefly refer about the literature data and summarize our personal experience in applying compression therapy in patients with mixed ulcers and moderate PAOD.

Despite the fact that arterial recanalization is often recommended as first therapeutical step in patients with leg ulcers and arterial impairment, ${ }^{2,3}$ there are some reports on clinical effectiveness of compression in favoring the healing of mixed ulcers with a not critical arterial disease. Ghauri ${ }^{4}$ applying compression in patients with mixed moderate arterial/venous ulcers achieved healing rates comparable with venous ulcers, although nurse-led surveillance was required. Humphreys ${ }^{5}$ managed patients with leg ulcers due to mixed moderate arterial/venous disease (ABPI above 0.5 up to 0.85 ) with supervised modified compression and considered for revascularization these patients only if their ulcer did not heal. He reported a $68 \%$ healing rate at 36 weeks in patients with mixed moderate arterial/venous disease and $87 \%$ in patients without arterial impairment. Georgopoulos $^{6}$ treated patients with moderate PAOD $(0.5<\mathrm{ABI}<0.75)$ conservatively with modified compression and considered patients with not-healing ulcers for revascularization. He reported a $64.7 \%$ healing rate at $24.7 \pm 3.1$ weeks with modified, reduced compression therapy. Marston reported two different studies on patient with PAOD treated just by compression without any surgical procedure for PAOD. In the first one ${ }^{7}$ he compared the outcomes of patients with mixed ulcers and moderate PAOD with patients affected by venous ulcers without any PAOD. He reported a fast healing time ( $57 \%$ of the ulcers healed at 10 weeks) although PAOD was a delaying factor. In the second paper he treated patients with pure PAOD ulcers ${ }^{8}$ and reported that compression therapy was effective even if the healing time was very slow ( $25 \%$ at 6 months and $52 \%$ at 12 months). Also in experimental setting compression was shown to be not harmful in patients with PAOD.

Top ${ }^{9}$ measured the toe pressure with and without short stretch bandages in patients with mixed leg ulcers and was able to show that compression did not significantly reduce toe pressure. Interface pressure was not measured. Our group ${ }^{10}$ reported that inelastic compression in patients with mixed and arterial ulcers does not reduce but even increase periwound flow, toe pressure and $\mathrm{TcPO}_{2}$ measured distally to the bandage provided that compression pressure is $<40 \mathrm{~mm} \mathrm{Hg}$ and that the perfusion pressure corresponding to the systolic ankle pressure is $>60 \mathrm{~mm} \mathrm{Hg}$.

Similar data were published by Junger, ${ }^{11}$ who reported that, after application of an inelastic multicomponent compression system in subjects with moderate PAOD, laser Doppler fluxmetry indicated significant improvements of the microcirculation.

Taking into consideration these data, we wanted to check the outcomes of patients with mixed ulcers with moderate PAOD treated by compression. We retrospectively analyzed the records of 180 outpatients (43 men and 137 females; mean age was $74 \pm 11.5$ years; age ranged between 31-92 years) with recalcitrant venous leg ulcers (VLU with ulcer surface up to $100 \mathrm{~cm}^{2}$ showing no healing tendency after a 6 months therapy) who had been treated between January 2011 and July 2014. In this case series we compared the clinical outcome of patients with mixed leg ulcers (MLU) with that of patients affected by pure VLU (pVLU). PAOD in mixed ulcers was considered moderate when ABPI was $<0.8$ and $>0.5$

Patients with small ulcers lower that $15 \mathrm{~cm}^{2}$ or lasting less than 6 months, which cannot be defined as recalcitrant were excluded, as also patients affected by severe PAOD with ABPI $<0.5$ who need to be referred for arterial revascularization as first therapeutical step.

Seventy-one out of 180 patients were affected by MLU with moderate PAOD and 109 by pVLU. All patients received the same local dressing (debridement with saline or light antiseptic solution and foam absorbing dressing). When clinical signs of infection occurred Cadexomer® powder was added until signs of infection disappeared. In addition to the same local wound dressing, the patients received foam sclerotherapy (sodium tetradecyl sulfate $3 \%$ ) of the superficial veins with reflux directed to the ulcer area and inelastic compression therapy (CT). In patients with pVLU CT was applied with a strong pressure higher than 60 $\mathrm{mm} \mathrm{Hg}$ and patients with MLU received short stretch bandages with a reduced pressure lower than $\leq 40 \mathrm{~mm} \mathrm{Hg}$. No patient was referred for arterial revascularization. The patients were followed until complete ulcer healing and healing rate was recorded.

Patients with MLU and pVLU showed comparable demographic characteristics in terms of sex, age, venous pathophysiology, ulcer surface, duration, wound-bed conditions and recurrence. Twenty-five patients were lost at follow-up and were excluded from final analysis. The outcomes were analyzed in the
Correspondence: Giovanni Mosti, Angiology Department, Barbantini Clinic, via del Calcio, 55100 Lucca, Italy.

E-mail: giovanni.mosti10@gmail.com

This work is licensed under a Creative Commons Attribution 4.0 License (by-nc 4.0).

(C) Copyright G. Mosti, 2016

Licensee PAGEPress, Italy

Veins and Lymphatics 2016; 5:5986

doi:10.4081/vl.2016.5986

remaining 155 patients [ 93 patients with pVLU (85.4\%) and 62 patients with MLU (87.4\%)].

The maximal time for complete healing was 48 weeks in pVLU group and 52 weeks in MLU $(\mathrm{P}=0.009)$. The median healing time of patients with pVLU (23 weeks) was significantly shorter than that of patients with MLU (25.5 weeks) ( $\mathrm{P}=0.03)$.

Multiple linear regression analysis showed the factors which influenced the healing time: deep venous disease $(\mathrm{P}<0.001)$, ulcer surface $(\mathrm{P}<0.001)$, arterial disease $(\mathrm{P}=0.002)$, and ulcer duration $(\mathrm{P}<0.01)$.

Pain ulcer-related rapidly disappeared. Pain cessation required an average of four weeks (in patients with VLU) and up to eight weeks in patients with mixed ulcers. Compression device-related pain or discomfort were minimal and tended to disappear when the patients got used to compression therapy.

In conclusion patients with mixed ulcers and moderate arterial disease may be effectively and safely treated with reduced, modified compression therapy characterized by short stretch material applied with reduced interface pressure $(<40 \mathrm{mmHg})$ and close surveillance. The vein disease treatment may be completed by foam sclerotherapy of refluxing veins. This treatment protocol may heal the mixed ulcers even if in a longer time period compared to pure VLU.

We suggest this conservative treatment protocol to patients with mixed ulcers not complicated by severe arterial disease restricting arterial revascularization procedures mainly to cases with critical ischemia (ABPI <0.5).

\section{References}

1. 0'Meara S, Cullum NA, Nelson EA. Compression for venous leg ulcers. Cochrane Database Syst Rev 2009;1: CD000265.

2. Lantis JC, Boone D, Lee L, et al. The effect of percutaneous intervention on wound healing in patients with mixed arterial venous disease. Ann Vasc Surg 2011;25:79-86. 
3. Hafner J, Schaad I, Schneider E, et al. Leg ulcers in peripheral arterial disease (arterial leg ulcers): impaired wound healing above the threshold of chronic critical limb ischemia. J Am Acad Dermatol 2000;43: 1001-8.

4. Ghauri AS, Nyamekye I, Grabs AJ, et al. The diagnosis and management of mixed arterial/venous leg ulcers in communitybased clinics. Eur J Vasc Endovasc Surg 1998;16:350-5.

5. Humphreys ML, Stewart AH, Gohel MS, et al. Management of mixed arterial and venous leg ulcers. $\mathrm{Br} \mathrm{J}$ Surg 2007;94:1104-7.
6. Georgopoulos S, Kouvelos GN, Koutsoumpelis A, et al. The effect of revascularization procedures on healing of mixed arterial and venous leg ulcers. Int Angiol 2013;32:368-74.

7. Marston WA, Carlin RE, Passman MA, et al. Healing rates and cost efficacy of outpatient compression treatment for leg ulcers associated with venous insufficiency. $\mathrm{J}$ Vasc Surg 1999;30:491-8.

8. Marston WA, Davies SW, Armstrong B, et al. Natural history of limbs with arterial insufficiency and chronic ulceration treated without revascularization. J Vasc Surg 2006;44:108-14.
9. Top S, Arveschoug AK, Fogh K. Do shortstretch bandages affect distal blood pressure in patients with mixed aetiology leg ulcers? J Wound Care 2009;18:439-42.

10. Mosti G, Iabichella ML, Partsch H. Compression therapy in mixed ulcers increases venous output and arterial perfusion. JVS 2012;55:122-8.

11. Junger M, Haase H, Schwenke L, et al. Macro- and microperfusion during application of a new compression system, designed for patients with leg ulcer and concomitant peripheral arterial occlusive disease. Clin Hemorheol Microcirc 2013;53:281-93. 